\title{
Flow Cytometry Identifies Risk Factors and Dynamic Changes in Patients with COVID-19
}

\author{
Daniele Moratto ${ }^{1} \cdot$ Marco Chiarini $^{1} \cdot$ Viviana Giustini $^{1,2} \cdot$ Federico Serana $^{3} \cdot$ Paola Magro $^{4} \cdot$ Aldo Maria Roccaro $^{2}$. \\ Luisa Imberti ${ }^{5}$. Francesco Castelli ${ }^{4}$. Luigi Daniele Notarangelo ${ }^{6}$ (D) Eugenia Quiros-Roldan ${ }^{4}$
}

Received: 22 May 2020 / Accepted: 16 June 2020 / Published online: 27 June 2020

(C) This is a U.S. Government work and not under copyright protection in the US; foreign copyright protection may apply 2020

Keywords COVID-19 $\cdot$ flow cytometry $\cdot$ T lymphocytes $\cdot$ monocytes $\cdot$ plasmablasts

\section{To the Editor:}

SARS-CoV-2 infection, first documented in Wuhan (China) at the end of 2019, rapidly spread to become a pandemic disease (COVID-19). The infection is highly contagious, and is characterized by a highly heterogeneous clinical phenotype, ranging from fully asymptomatic to severe disease and a high fatality rate [1]. While the reasons for such phenotypic heterogeneity remain ill-defined, some patterns have emerged. In particular, in individuals with severe clinical course, the disease often manifests in two waves [2]. In the first phase, patients present typical clinical symptoms of a respiratory tract infections, whereas in the second phase (approximately 8-12 days after onset of symptoms), sudden worsening of respiratory status occurs. At this stage, diffuse ground-glass opacities are observed on chest computed tomography. Lymphopenia, elevated D-dimer levels, and prolonged prothrombin time are common laboratory abnormalities in these critically ill patients [2,3]. Although the infection has been documented in subjects of all ages, the

Daniele Moratto and Marco Chiarini contributed equally to this work.

Electronic supplementary material The online version of this article (https://doi.org/10.1007/s10875-020-00806-6) contains supplementary material, which is available to authorized users.

Luigi Daniele Notarangelo

luigi.notarangelo2@nih.gov

Eugenia Quiros-Roldan

eugeniaquiros@yahoo.it

1 Flow Cytometry Unit, Clinical Chemistry Laboratory, ASST Spedali Civili di Brescia, Brescia, Italy

2 Clinical Research Development and Phase I Unit, ASST Spedali Civili di Brescia, Brescia, Italy mortality rate is particularly high among elderly ( $>70$ years) individuals. Pre-existing co-morbidities (such as diabetes, hypertension, and chronic lung disease) are associated with worse outcome.

The mechanisms underlying worsening of the disease are object of intense investigation. Increased levels of interleukin-6 (IL-6) and tumor necrosis factor- $\alpha$ (TNF- $\alpha$ ) have been documented in severely infected individuals [2-4]. Along with lymphopenia, these observations have led to hypothesize that dysregulation of immune responses may play an important role in the pathology of the disease. However, limited data are available on the immune status of COVID-19 patients. In particular, it remains unknown whether the study of immune parameters at the time of admission to the hospital may help distinguish those who will progress to respiratory failure and will require admission to intensive care units (ICU) and possibly die, from those with moderate symptoms, who will not require invasive ventilation and will eventually recover.
3 Hematology Unit, Clinical Chemistry Laboratory, ASST Spedali Civili di Brescia, Brescia, Italy

4 Department of Infectious and Tropical Diseases, University of Brescia and ASST Spedali di Brescia, Brescia, Italy

5 CREA Laboratory, ASST Spedali Civili di Brescia, Brescia, Italy

6 Laboratory of Clinical Immunology and Microbiology, National Institute of Allergy and Infectious Diseases, National Institutes of Health, Building 10, room 5-3950, 10 Center Drive, Bethesda, MD 20892, USA 
We hypothesized that peripheral blood mononuclear cell (PBMC) phenotypic abnormalities detected shortly after hospitalization may help identify patients at higher risk of worse outcome. To test this hypothesis, we collected peripheral blood samples from 36 COVID-19 patients who have been admitted to the Infectious Disease Unit of the Spedali Civili, Brescia (Italy), a northern Italy city where a large fraction of the population has suffered from SARS-CoV-2 infection and where a high mortality rate has been documented [5]. Blood was obtained upon informed consent and according to a protocol approved by the local ethical committee. Details of the clinical and laboratory abnormalities of the patients are reported in Supplementary Tables 1 and 2. The interval between onset of symptoms and admission to the hospital was $7.78 \pm$ 0.65 (mean \pm SEM) days. Disease severity was assessed according to the Sixth Revised Trial Version of the Novel Coronavirus Pneumonia Diagnosis and Treatment Guidance. Of the 36 patients, eight manifested a moderate disease phenotype (patients P1 to P8 in Supplementary Tables 1 and 2), and 28 a severe phenotype at admission. All those with a moderate phenotype recovered and were eventually discharged $6.62 \pm 1.39$ days since admission. Among the 28 patients with a severe phenotype, 22 (patients $\mathrm{P} 9$ to $\mathrm{P} 30$ in Supplementary Tables 1 and 2) gradually improved and were discharged $13.68 \pm 1.04$ days since admission, whereas the remaining six patients (P31 to P36 in Supplementary Tables 1 and 2) progressed to critical clinical conditions. Four of these died, and two were transferred to the ICU. Of the latter, one recovered and was discharged 26 days since admission, while the other one is still in the hospital. Analysis of the PBMC phenotype in the entire cohort was performed $2.78 \pm 0.45$ days after admission; moreover, longitudinal values were recorded in 26 of the 36 patients, in the attempt to document dynamic fluctuations of the immune profile during evolution towards recovery.

Flow cytometry analysis at the time of first evaluation after admission revealed significant differences among patients with moderate disease, those with a severe phenotype who eventually recovered, and those who progressed to a critical phenotype (Fig. 1). In particular, the absolute counts of $\mathrm{CD}^{+}, \mathrm{CD}^{+}$, and $\mathrm{CD} 8^{+}$lymphocytes at admission were the variables that distinguished more clearly among the three groups of patients. Furthermore, patients with severe disease had a significantly reduced number of TCR $\gamma \delta^{+} \mathrm{T}$ cells, and an abnormal distribution of $\mathrm{CD} 8^{+} \mathrm{T}$ cell subsets, with lower proportion of naïve cells and increased percentage of effector CD45RA ${ }^{+}$cells (Fig. 1). No significant differences were observed in the proportion of the other $\mathrm{T}$ cell subsets and in the count of natural killer cells (Supplementary Fig. 1). Comparison of the three groups of patients also showed a lower count of circulating B cells in those with severe disease, while a significantly increased proportion of circulating $\mathrm{CD} 19^{+} \mathrm{CD} 20^{-} \mathrm{CD} 38^{\mathrm{hi}} \mathrm{CD} 27^{\mathrm{hi}}$ plasmablasts appeared as a common characteristic of all patients, irrespective of the severity of the disease (Fig. 1). An early specific antibody response, with a significant IgG component, has been documented in patients with COVID-19. ${ }^{6}$ It has been previously shown that non-neutralizing IgG antibodies produced during SARS facilitated internalization of virusantibody immune complexes in myeloid cells, promoting inflammation and tissue injury, a phenomenon referred to as antibody-dependent enhancement. High titers of anti-N and anti-S IgG and IgM antibodies have been associated by others with a more severe clinical outcome also in patients with COVID-19, especially if of older age [6, 7]. Whether this reflects occurrence of antibody-dependent enhancement also in COVID-19 remains to be seen.

It has been recently shown that progression to severe and critical disease in patients with COVID-19 is associated with reduced expression of HLA-DR molecules on the surface of monocytes, and it has been speculated that this immune abnormality may underlie inadequate adaptive immune responses due to impaired SARS-CoV-2 antigen presentation [8]. Our results confirm these findings. Such abnormalities of monocyte phenotype are in contrast with what observed in HIV-infected patients, in whom increased HLA-DR expression on the surface of monocytes is associated with apoptosis and depletion of $\mathrm{CD}^{+} \mathrm{T}$ cells, and can be reversed by antiretroviral therapy $[9,10]$. Importantly, our results indicate that measurement of HLA-DR and CD4 mean fluorescent intensity (MFI) on the surface of monocytes at admission may not only distinguish patients with moderate versus those with severe disease but may also identify patients with severe phenotype who will progress to a critical status versus those who will improve and eventually recover (Fig. 1).

By the time of the last evaluation before discharge, patients who attained clinical recovery manifested significant improvement of several immunological parameters. However, multilevel mixed linear regression analysis of longitudinal values collected during hospitalization demonstrated that the three groups of patients remained clearly distinct for a number of parameters. In particular, while progressive improvement in the counts of $\mathrm{CD}^{+}, \mathrm{CD}^{+}$, and $\mathrm{CD} 8^{+}$cells was observed in patients with moderate or severe disease who eventually recovered, those who progressed to a critical status remained with very low counts of all of these subsets, associated with markedly reduced expression of HLA-DR and CD4 molecules on the surface of monocytes (Supplementary Fig. 2). Longitudinal analysis of B lymphocyte subset distribution demonstrated that the proportion of $\operatorname{IgM}^{+}$and switched memory B cells progressively increased in most patients, in particular 2 weeks after onset of symptoms, whereas the proportion of plasmablasts concomitantly decreased and generally normalized by the day of discharge (Supplementary Fig. 3).

Although the low number of patients and the possible confounding effects of co-morbidities and of various therapeutic modalities used represent obvious limitations of our study, we 


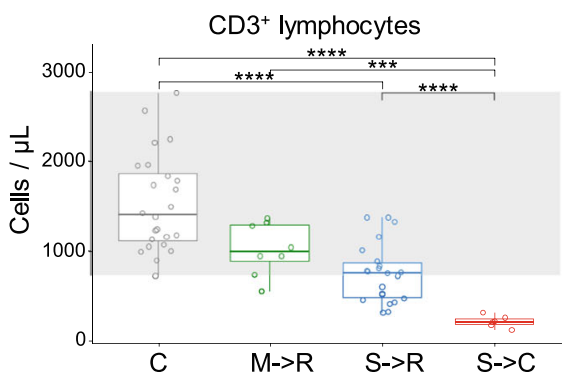

CD4 ${ }^{+}$CD $45 R^{+}{ }^{+}$CCR7 $7^{+}$lymphocytes
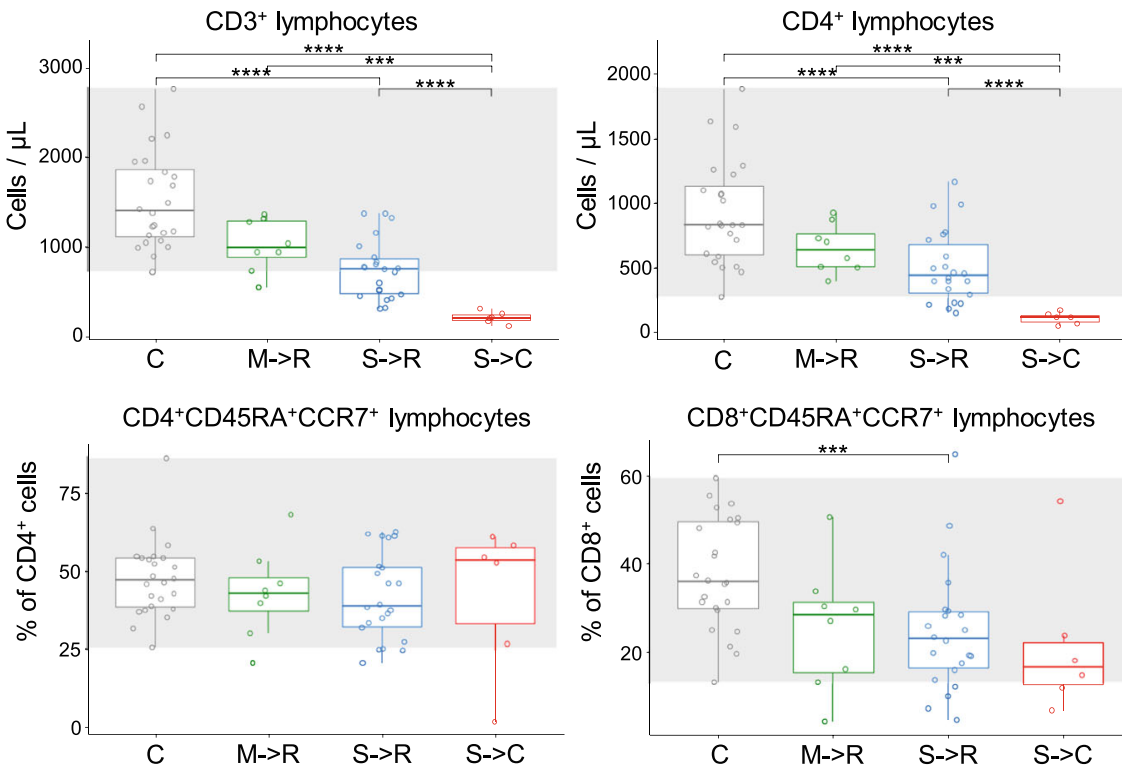

$\mathrm{CD}^{+} \mathrm{CD} 45 \mathrm{RA}{ }^{+} \mathrm{CCR} 7^{+}$lymphocytes
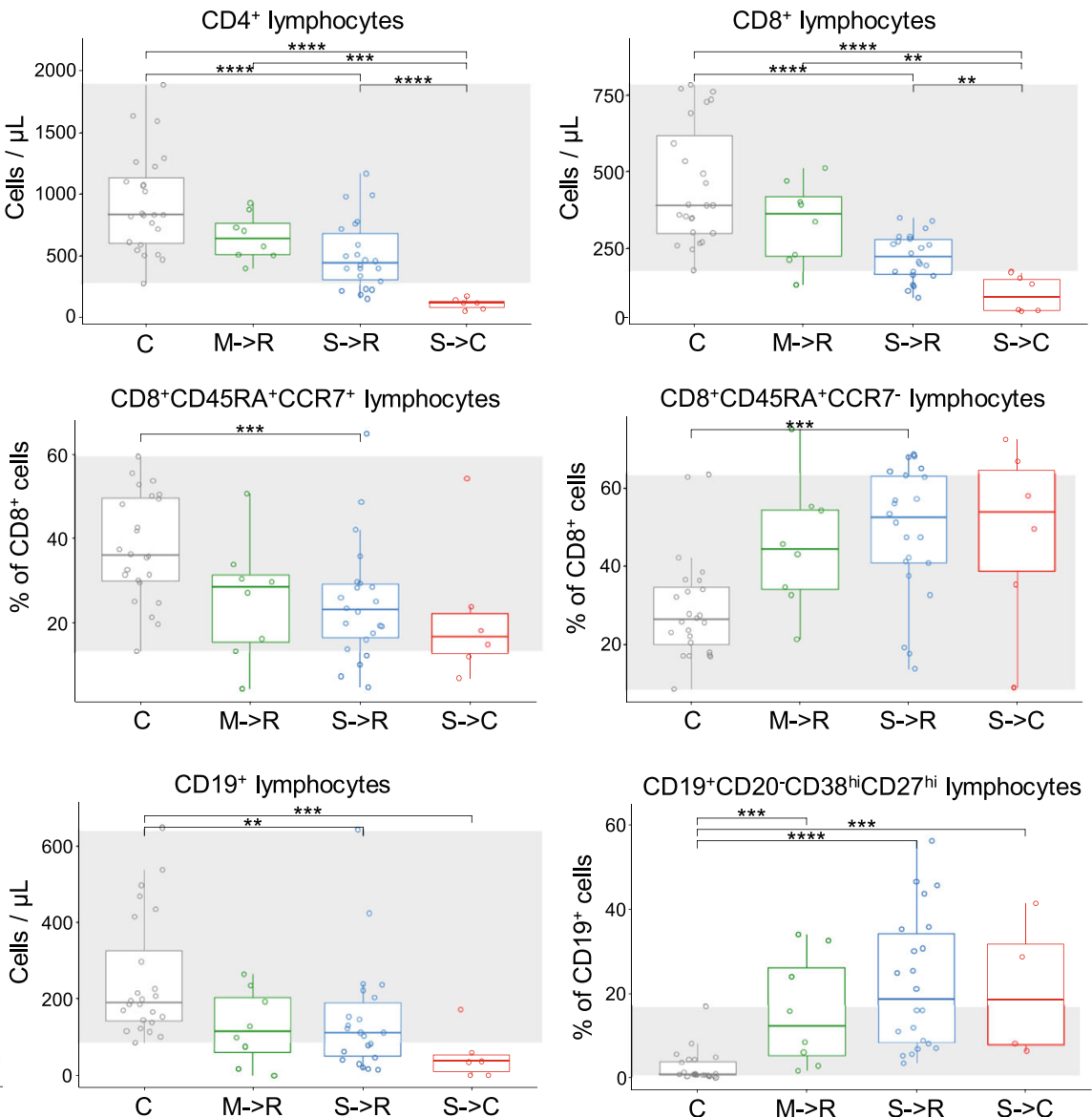

CD8 ${ }^{+}$CD45RA ${ }^{+}$CCR7- lymphocytes

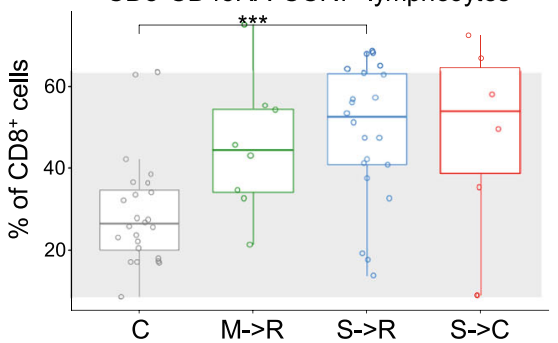

CD19+CD20-CD38 ${ }^{\text {hi }} C D 27^{\text {hi }}$ lymphocytes

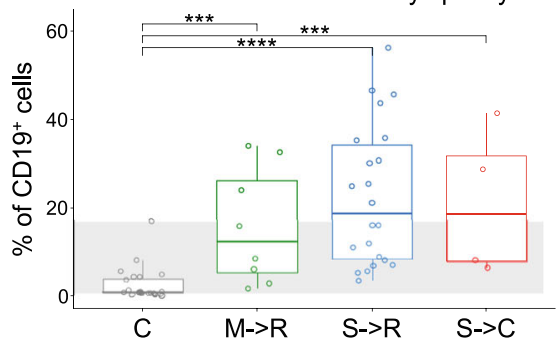

HLA DR expression on monocytes
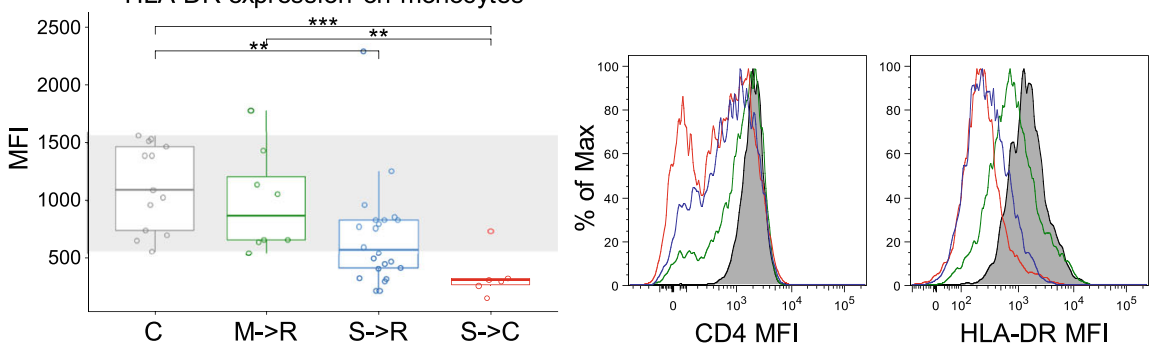

Fig. 1 Lymphocyte and monocyte phenotype at first evaluation. Comparison of lymphocytes subsets (upper rows) and surface marker expression on monocytes (bottom row) at the time of first evaluation between controls (C) and COVID-19 patients classified as moderate who recovered $(\mathrm{M}->\mathrm{R})$, severe who recovered $(\mathrm{S}->\mathrm{R})$, and severe

who progressed to critical status $(\mathrm{S}->\mathrm{C})$. The shaded area represents the range of normal values in controls. ** $p<0.01$, $* * * p<0.001, * * * * p<0.0001$. Pairwise comparisons were made by the Mann-Whitney test with Bonferroni correction, after a significant Kruskal-Wallis test

were able to detect correlation between some of the immunological abnormalities observed and quantitative measures of disease severity. In particular, Kaplan-Meyer analysis demonstrated that lower $\mathrm{CD}^{+}$cell count and reduced CD4 MFI expression on the surface of monocytes at the time of first evaluation after admission were associated with increased duration of the disease (Supplementary Fig. 4, panel A). Moreover, linear regression analysis showed that among survivors, lower counts of CD3, CD4, and CD8 cells; lower CD4 and HLA-DR MFI on the surface of monocytes; and higher levels of CRP, ferritin, and LDH at admission were associated with prolonged duration of hospitalization (Supplementary Fig. 4, panel B).

Finally, our patient cohort included also a male (P2) affected with $\mathrm{X}$-linked agammaglobulinemia, a genetic condition due to mutations of the Bruton's tyrosine kinase (BTK) gene. The moderate COVID-19 phenotype manifested by this patient is consistent with the promising results that have been recently reported in a series of 19 patients with severe COVID-19 who have received acalabrutinib, a BTK inhibitor [11]. 
In conclusion, our data suggest that flow cytometry analysis of PBMC phenotype may help predict the risk of clinical progression of COVID-19 and indicate that recovery from COVID-19 is accompanied by improved antigen presentation and more effective adaptive immune responses.

Acknowledgements This work was supported by Regione Lombardia, Italy (project "Risposta immune in pazienti con COVID-19 e comorbidita"'). L.D.N. is supported by the Division of Intramural Research, National Institute of Allergy and Infectious Diseases, National Institutes of Health, USA.

\section{Compliance with Ethical Standards}

Conflict of Interest The authors declare that they have no conflict of interest.

Ethical Statement The study was approved by Comitato Etico Provinciale. Blood samples were obtained as part of the patients' clinical evaluation.

\section{References}

1. Guan WJ, Ni ZY, Hu Y, Liang WH, Ou CQ, He JX, et al. Clinical characteristics of coronavirus disease 2019 in China. N Engl J Med. 2020;382:1708-20.

2. Huang C, Wang Y, Li X, Ren L, Zhao J, Hu Y, et al. Clinical features of patients infected with 2019 novel coronavirus in Wuhan, China. Lancet. 2020;395:497-506.

3. Zhou F, Yu T, Du R, Fan G, Liu Y, Liu Z, et al. Clinical course and risk factors for mortality of adult inpatients with COVID-19 in
Wuhan, China: a retrospective cohort study. Lancet. 2020;395: 1054-62.

4. Hadjadj J, Yatim N, Barnabei L, Corneau A, Boussier J, Pere H, et al. Impaired type I interferon activity and exacerbated inflammatory responses in severe Covid-19 patients. medRxiv 2020. https:// doi.org/10.1101/2020.04.19.20068015.

5. Piva S, Filippini M, Turla F, Cattaneo S, Margola A, De Fulviis S, et al. Clinical presentation and initial management critically ill patients with severe acute respiratory syndrome coronavirus 2 (SARS-CoV-2) infection in Brescia, Italy. J Crit Care. 2020;58: 29-33.

6. Long QX, Liu BZ, Deng HJ, Wu GC, Deng K, Chen YK, et al. Antibody responses to SARS-CoV-2 in patients with COVID-19. Nat Med. 2020;29.

7. Tan W, Lu Y, Zhang J, Dan Y, Tan Z, He X, et al. Viral kinetics and antibody responses in patients with COVID-19. medRxiv 2020. https://doi.org/10.1101/2020.03.24.20042382.

8. Giamarellos-Bourboulis EJ, Netea MG, Rovina N, Akinosoglou K, Antoniadou A, Antonakos N, et al. Complex immune dysregulation in COVID-19 patients with severe respiratory failure. Cell Host Microbe. 2020;17.

9. Gascon RL, Narváez AB, Zhang R, Kahn JO, Hecht FM, Herndier BG, et al. Increased HLA-DR expression on peripheral blood monocytes in subsets of subjects with primary HIV infection is associated with elevated CD4 T-cell apoptosis and CD4 T-cell depletion. J Acquir Immune Defic Syndr. 2002;30:146-53.

10. McCausland MR, Juchnowski SM, Zidar DA, Kuritzkes DR, Andrade A, Sieg SF, et al. Altered monocyte phenotype in HIV-1 infection tends to normalize with integrase-inhibitor-based antiretroviral therapy. PLoS One. 2015;10:e0139474.

11. Roschewski M, Lionakis MS, Sharman JP, Roswarski J, Goy A, Monticelli MA, et al. Inhibition of Bruton tyrosine kinase in patients with severe COVID-10. Sci Immunol. 2020;5:eabd0110.

Publisher's Note Springer Nature remains neutral with regard to jurisdictional claims in published maps and institutional affiliations. 